\title{
Vertical and horizontal abundance structures of the roAp star HD 24712
}

\author{
T. Lüftinger ${ }^{1}$, O. Kochukhov ${ }^{1}$, T. Ryabchikova ${ }^{1,3}$, I. Ilyin ${ }^{2}$ \\ and W.W. Weiss ${ }^{1}$ \\ ${ }^{1}$ Department of Astronomy, Vienna University, Tuerkenschanzstrasse 17, A-1180 Vienna, \\ Austria, email: name@astro.univie.ac.at \\ ${ }^{2}$ Astrophysical Institute Potsdam, An der Sternwarte 16, D-14482 Potsdam, Germany \\ email: ilyin@aip.de \\ ${ }^{3}$ Institute for Astronomy, Russian Academy of Sciences, Pyatnitskaya 48, 109017 Moscow, \\ Russia, email: ryabchik@inasan.rssi.ru
}

\begin{abstract}
High-resolution spectroscopic and spectropolarimetric data of the rapidly oscillating Ap star HD 24712 (HR 1217, DO Eri) has been analysed including modelling the vertical elemental abundance structures. We study the interaction and the relation of the vertical (stratification) and the horizontal (spots) abundance characteristics of $\mathrm{Fe}$ and the stellar magnetic field. By this synopsis and the relation of our results to the analysis of high resolution and high time resolved observations (Sachkov et al. 2005) we are likely to gain new insights about the atmospheric structure and the geometry, the origin, and the evolution of the magnetic fields of roAp stars.
\end{abstract}

Keywords. Stars: abundances, stars: stratification, stars: imaging, stars: individual: (HD 24712)

\section{Introduction}

HD 24712 (HR 1217, DO Eri, $V=6.00$ ) is an intensively studied roAp star with light (Wolff \& Morrison 1973), spectrum and magnetic variations. Kurtz (1981), after having discovered photometric oscillations of $6.15 \mathrm{~min}$, found that the maximum of the pulsational amplitude of this star corresponds to the maximum of the longitudinal magnetic field (Kurtz 1982). Matthews et al. (1988) discovered radial velocity variations with the main photometric period and an amplitude of $0.4 \pm 0.05 \mathrm{~km} \mathrm{~s}^{-1}$. Ryabchikova et al. (2000) detected the greatest line intensity variations for the REE, and found that the region of REE overabundances roughly coincides with the visible magnetic pole. According to their analysis and ours (Lueftinger et al. 2003) Fe varies in antiphase and with a smaller amplitude and seems to be accumulated in a ring-like structure around the magnetic equator. Evidence was found for element stratification in the atmosphere of this star.

\section{Observations and fundamental parameters}

High resolution $(R=80000)$ spectroscopic and spectropolarimetric observations were obtained with the SOFIN spectrograph attached to the Northern Optical Telescope (NOT) in November 2003. One spectrum obtained with the VLT UVES spectrograph at ESO was obtained near the magnetic minimum of the star. It is from the UVES POP database (Bagnulo et al. 2005). Using the new stellar model atmosphere code LLModels (Shulyak et al. 2004), which implements a direct accounting for line opacities, we obtained the best fit to the $\mathrm{H} \alpha$ line with $T_{\text {eff }}=7350 \mathrm{~K} \cdot \log g=4.3$ was used as determined by Ryabchikova et al. (1997). 

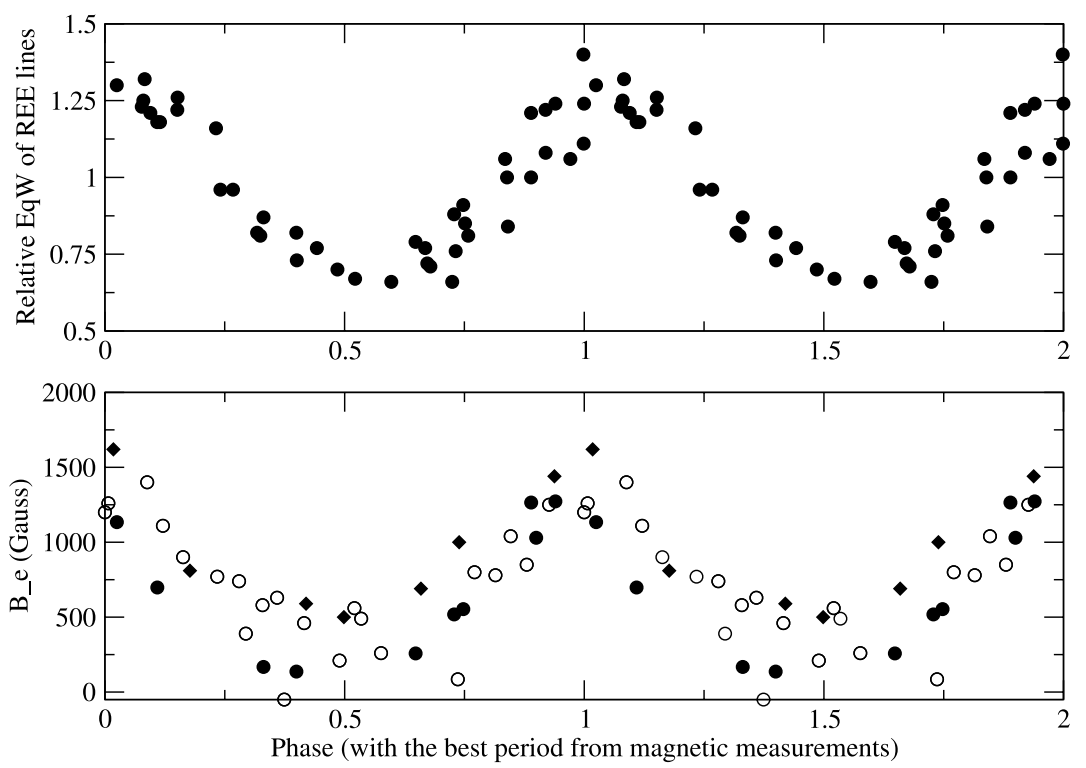

Figure 1. Magnetic field measurements of HD 24712 phased with the best fit period of $12.45965 \pm 0.00020 \mathrm{~d}$.

\section{Rotational period}

The rotational period of HD 24712 is an ongoing quest: Preston (1972) derived a period of $P=12.448 \mathrm{~d}$, but Bonsack (1979) needed a slightly longer period $12.46 \mathrm{~d}$ to phase his data with that of Preston. Kurtz \& Marang (1987) combined their high-speed photometry data and that of Wolff \& Morrison (1987), which led to an improved period of $12.4572 \pm 0.0003 \mathrm{~d}$, but the extrema of photometric and magnetic variations were then separated. Catalano et al. (1991) obtaining photometric observations in the JHK bands and again found a phase shift between the photometric and the magnetic variations. Mathys (1991), combining various measurements of the mean longitudinal magnetic field, derived a period of $P=12.4610 \pm 0.0025 \mathrm{~d}$, which agrees with that of Bagnulo et al. (1995) $P=12.4610 \pm 0.0011$ d. Combining measurements of Preston (1972), Mathys and Landstreet, and MuSiCoS (e.g., Wade et al. 2000), we obained a rotational period of $12.45965 \pm 0.00020 \mathrm{~d}$. Our search for an improved rotational period has been performed using the program Period98 (Sperl 1998). The highest peak in the amplitude spectrum is used to specify the starting frequency and amplitude for a least-squares fit yielding the period, the amplitude and the phase angle. The corresponding rms errors are the results of numerical simulations of error propagation as provided by the program EPSim (Reegen 2004).

\section{Stratification analysis}

Peculiar A stars, such as HD 24712, usually posess a global magnetic field, which stabilize their atmospheres and enables the mutual diffusion of the chemical elements due to different separation processes. Calculations of self consistent model atmospheres including the effect of diffusion, predict changing abundance profiles for a large number of elements and the corresponding changes in the atmospheric structure. Thus, if we want to derive the surface abundances and the magnetic field geometry of Ap and roAp stars, it is indispensible to account for stratification effects within the atmosphere, which 


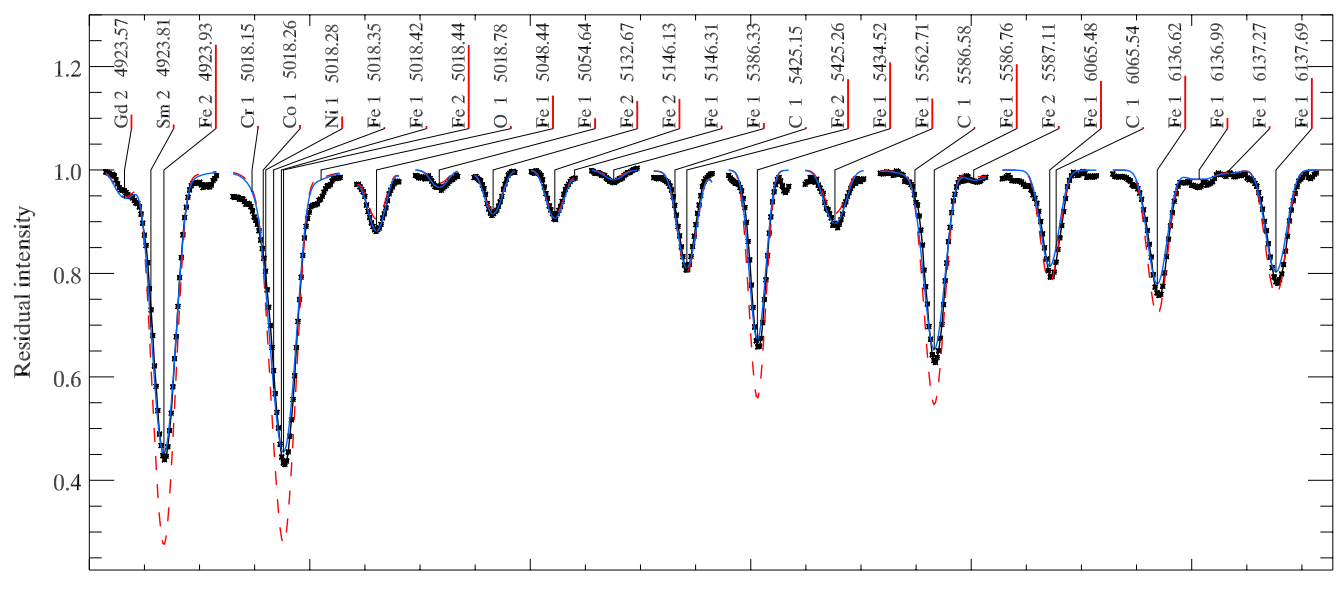

Wavelength

Figure 2. Comparison between observed and calculated line profiles with homogeneous abundances (dashed line) and with stratified abundance distribution (full line) around magnetic minimum.

are also shown in Fig. 5, where we compare model calculations of Intensity- and Stokes profiles of the Fe II line near $5018 \AA$ with and without stratification. Observations that require us to account for the vertical chemical abundance inhomogeneities are

- The impossibility to fit the spectral line wings and the core with the same abundance

- Different abundance values for one element derived from different ionization stages

- Disagreement of the abundances derived from strong and weak lines of the same element

- Unusual behaviour of very high-excitation lines

For HD 24712 we studied the abundance stratification for Fe using DDAFIT, a newly developed procedure for the determination of vertical abundance gradients written in

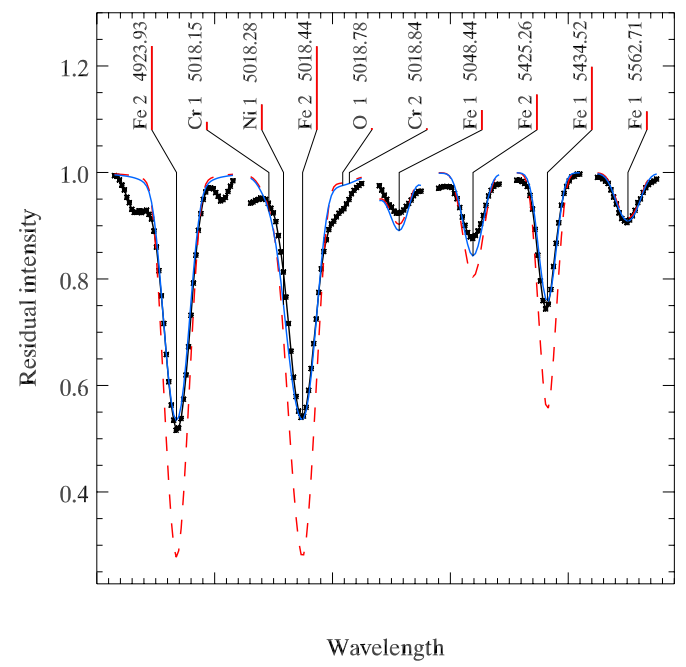

Figure 3. Comparison between observed and calculated line profiles with homogeneous abundances (dashed line) and with stratified abundance distribution (full line) around magnetic maximum. 


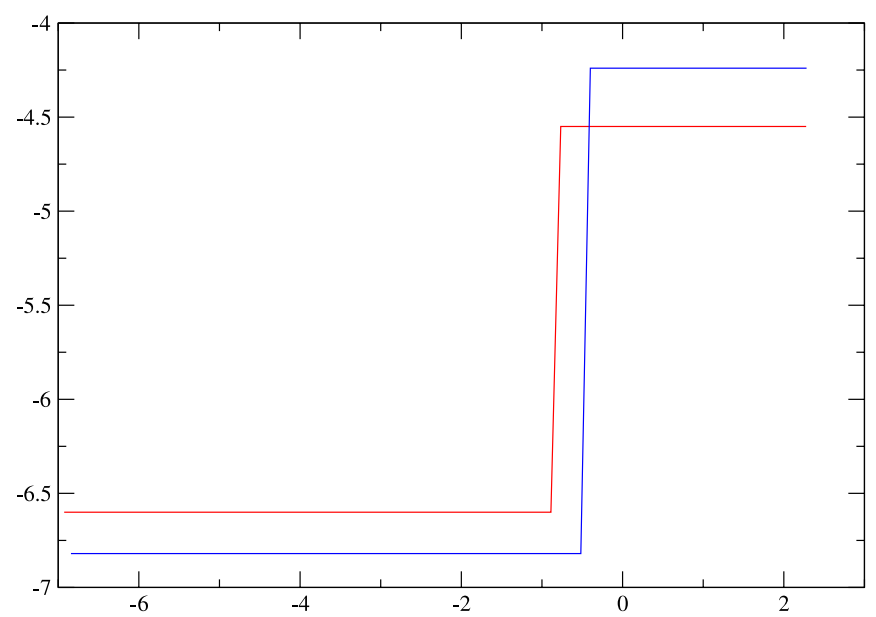

Figure 4. Abundance stratification of Fe in the atmosphere of HD 24712. Abundances in dex are plot versus atmospheric depth in $\log \tau_{5000}$.

IDL by Kochukhov, providing an optimization and visualization interface to the spectrum synthesis calculations with SYNTH3, a modification of SYNTH (Piskunov 1992). Vertical abundance distributions were derived using a one step model. The chemical abundance in the upper atmosphere, the abundance in deeper layers, and the vertical position of the abundance step were optimized simultaneously by least squares fitting. With the high resolution spectra obtained around magnetic minimum of the star (UVES) and around magnetic maximum (NOT), we derived stratification profiles for both phases. For the phase around magnetic maximum only six Fe lines could be used and the results can only be regarded as a first approach. In Fig. 2 and Fig. 3 a comparison between observed and calculated profiles of spectral lines selected for the stratification analysis is given. The homogeneous (dashed line) and the stratified (full line) abundances are presented. Our analysis, as presented in Fig. 4, reveals a change in the Fe abundance from $-6.6 \pm 0.046$ dex in the higher atmospheric layers to $-4.55 \pm 0.022$ below $\log \tau_{5000}=-0.7$ around the phase of magnetic minimum (dotted line). Observations around magnetic maximum (full line) suggest the abundance jump occurs in slightly deeper atmospheric layers, around $\log \tau_{5000}=-0.5$.

\section{Conclusions and the future}

HD 24712 is the first star, where an analyses of the elemental surface abundances and the magnetic field geometry is being performed, including and accounting for effects of the changing vertical abundance within the stellar atmosphere. It is now possible to simultaneously reconstruct the chemical distribution and the magnetic field geometry on the stellar surface (INVERS10, Piskunov et al. 2002) including the detailed analysis of the vertical abundance gradients. To reliably determine the elemental surface abundance patterns of various chemical elements on the surface of HD 24712, we will derive the stratification profiles of additional (iron-peak) elements, and further investigate the analysis of the Fe stratification profile around the magnetic maximum of the star, where it seems that the abundance step is moved towards deeper atmospheric layers. Observing a larger sample of Ap and roAp stars in all four Stokes parameters and combining the analysis 


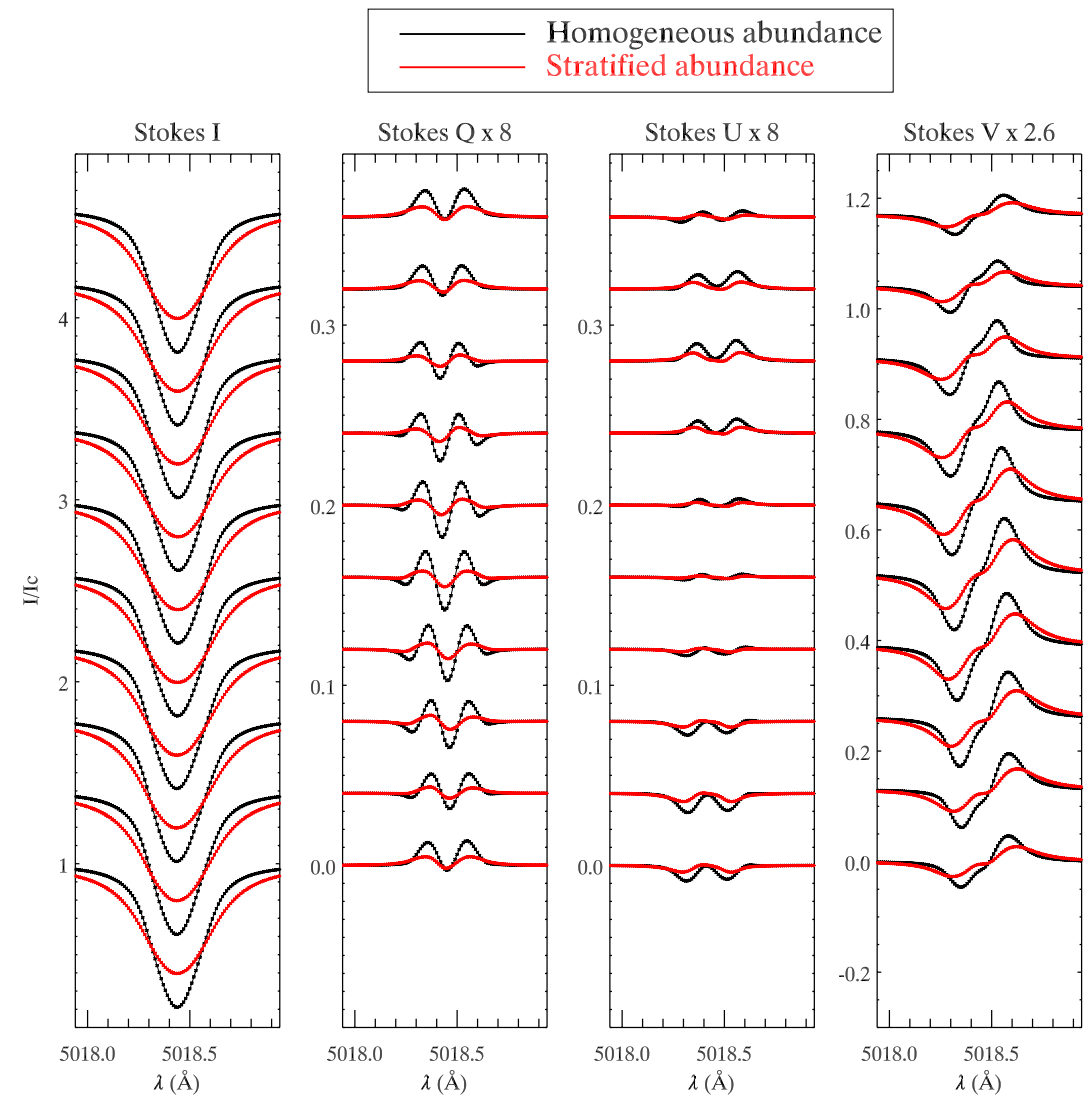

Figure 5. Intensity and Stokes profiles of the Fe II line near $5018 \AA$.

of the vertical and the horizontal abundance gradients and the magnetic field geometry, we expect to gain new insights into the atmospheric structure of Ap and roAp stars.

\section{Acknowledgements}

Financial support for this research was received from the Austrian 'Fonds zur Förderung der wissenschafltichen Forschung' (projects P14984 and P14546).

\section{References}

Bagnulo, S., Landi degl'Innocenti, E., Landolfi, M., Leroy, J. L. 1995, A\&A 295, 459

Bagnulo, S., Jehin, E., Melo, C., Ledoux, C., Cabanc, R. 2005, These Proceedings, JP6

Bonsack, W.K. 1979, PASP 91, 648

Catalano, F.A., Kroll, R., Leone, F. 1991, A $\& A$ 248, 179

Lueftinger, T., Ryabchikova, T.A., Kochukhov, O., Piskunov, N.E., Kuschnig, R., Wade, G.A., Weiss, W.W. 2003, International Conference on magnetic fields in $O, B$, and $A$ stars, ASP Conference Series, Vol. No. 305, L.A. Balona, H.F. Henrichs, 83 R. Medupe, eds. 305, 92 Kochukhov, O., Piskunov, N. 2002, A\&A 388, 868

Kurtz, D.W. 1981, Inform. Bull. Var. Stars 1915, 1

Kurtz, D.W. 1982, MNRAS 200, 807

Mathys, G., 2000, AESAS 89, 121

Martinez, P., Girish, V., Joshi, S., Kurtz, D. W., Ashoka, B. N.,Chaubey, U. S., Gupta, S. K., Sagar, R. 2000, IBVS 4853 
Martinez, P., Kurtz, D.W., Ashoka, B.N., et al. 2001, A\&A 371, 1048

Matthews, J.M., Wehlau, W.H., Walker, G.A.H., Yang, S. 1988, ApJ 324, 1099

Piskunov, N.E., 1992, in Glagolevskij Yu. V., Romanyuk I.I., eds., Stellar Magnetism. Nauka, St. Petersburg 92

Piskunov, N.E., Kochukhov, O. 2002, A\&A 381, 736

Preston G.W. 1972, ApJ 175, 465

Reegen, P. 2004, In: Second Eddington Workshop: Stellar structure and habitable planet finding, 9 - 11 April 2003, Palermo, Italy. Edited by F. Favata, S. Aigrain and A. Wilson. ESA SP-538, Noordwijk: ESA Publications Division, ISBN 92-9092-848-4 389

Ryabchikova, T.A., Landstreet, J.D., Gelbmann, M.J., Bolgova, G.T.,Tsymbal, V.V., Weiss, W.W. 1997, A\&A 327, 1137

Sachkov, M., Ryabchikova, T., Ilyin, I., Kochukhov, O., Lueftinger, T. 2005, These Proceedings, GP2

Shulyak, D., et al. 2004, A\& $A$ accepted

Sperl, M. 1998, CoAst. 111, 1

Wade, G.A., Donati, J.-F., Landstreet, J.D., Shorlin, S.L.S. 2000, MNRAS 313, 851,

Wade, G.A., Bagnulo, S., Donati, J.-F., Lueftinger, T., Petit, P., Sigut, T.A.A. 2001, APN 35

Wolff, S.C., Morrison N.D. 1973, PASP 85, 141

\section{Discussion}

Mkrtichian: Fe lines in HR 1217 show small intensity variations over the rotation period. Can you show the results of stratification analyses for lines that show a strong intensity variation over the rotation period?

LUEFTINGER: So far, we derived stratification profiles for Fe, but indeed, stratification analysis for further elements, of course and especially including lines with strong intensity variations, is one of the very next steps in our investigation.

WeIss: You showed two stratification profiles for minimum and maximum magnetic field. Can you please comment comment on the error bar?

LuEfTINGER: The error bar of abundances are below \pm 0.05 dex. The abundance jump was determined with an accuracy of \pm 0.009 in rhox

MONIER: How accurate is the location of the optical depth $\left(\log \tau_{5000}\right)$ at which the abundance discontinuity occurs in your analysis of HD 24712 ?

KochuKHOv: The accuracy of the derivation of the position of vertical abundance jump is about 0.1 dex in the $\log \tau_{5000}$ scale.

BAgnulo: You have adopted a step function to approximate the stratification of the chemical elements. Is that real? Have you tried to use for instance a second order polynomial?

LUEFTINGER: It is possible to apply, e.g., second order polynomials, and also vary the width of the transition region, but we found that in most cases a one step model is a very good and evidently realistic approximation.

Ryabchikova, Remark to Bagnulo's question: Many experiments with stratification calculations found that steep abundance jumps (about zero width of abundance transition zone) frequently occur in Ap atmospheres. However, wider transition zones seems to be needed in the case of strongly magnetic stars, not excluding a polynomial shape of abundance transition zone. 